\title{
Ensino da medida da pressão arterial entre hipertensos: estratégia educativa com simulador de baixa fidelidade
}

Teaching blood pressure measurement among hypertensive patients: educational strategy with low-fidelity simulator Enseñanza de la medición de la presión arterial en pacientes hipertensos: estrategia educativa con simulador de baja fidelidade

\section{RESUMO}

Objetivo: Avaliar o conhecimento prático sobre a técnica da medida indireta da pressão arterial realizada por pacientes hipertensos no domicílio antes e após intervenção educativa com simulador de baixa fidelidade. Métodos: Estudo quase-experimental, do tipo antes e depois, realizado em uma Unidade de Estratégia de Saúde da Família, no período de janeiro a julho de 2016. A amostra de conveniência foi composta por 30 hipertensos, maiores de 18 anos, que utilizaram um boneco articulado de madeira como simulador de baixa fidelidade para reproduzir as etapas da medida indireta da pressão arterial no domicílio. Resultados: Após a estratégia educativa os hipertensos apresentaram maior proporção de acertos em relação ao preparo do ambiente, preparo do paciente e posicionamento do corpo. Conclusão: o uso de um simulador de baixa fidelidade teve impacto positivo na promoção do conhecimento sobre a técnica de medida indireta da pressão arterial no domicílio.
\end{abstract}

DESCRITORES: Pressão Arterial, Determinação da Pressão Arterial, Educação em Enfermagem, Materiais de Ensino, Simulação.

\section{ABSTRACT}

Objective: To evaluate practical knowledge about the technique of indirect blood pressure measurement performed by hypertensive patients at home before and after educational intervention with a low-fidelity simulator. Methods: Quasi-experimental study, of the before and after type, carried out in a Family Health Strategy Unit, from January to July 2016. The convenience sample consisted of 30 hypertensive patients, over 18 years old, who used an articulated wooden doll as a low-fidelity simulator to reproduce the steps of indirect blood pressure measurement at home. Results: After the educational strategy, the hypertensive patients had a higher proportion of correct answers in relation to the preparation of the environment, preparation of the patient and positioning of the body. Conclusion: the use of a low-fidelity simulator had a positive impact on promoting knowledge about the technique of indirect blood pressure measurement at home.

DESCRIPTORS: Arterial Pressure, Blood Pressure Determination, Education, Nursing, Teaching Materials, Simulation Techinique.

\section{RESUMEN}

Objetivo: Evaluar los conocimientos prácticos sobre la técnica de medición indirecta de la presión arterial realizada por pacientes hipertensos en su domicilio antes y después de la intervención educativa con un simulador de baja fidelidad. Métodos: estudio cuasiexperimental, del tipo antes y después, realizado en una Unidad de Estrategia de Salud de la Familia, de enero a julio de 2016. La muestra de conveniencia estuvo conformada por 30 pacientes hipertensos, mayores de 18 años, quienes utilizaron un muñeco de madera articulado como un simulador de baja fidelidad para reproducir los pasos de la medición indirecta de la presión arterial en casa. Resultados: Luego de la estrategia educativa, los pacientes hipertensos tuvieron una mayor proporción de respuestas correctas en relación a la preparación del ambiente, preparación del paciente y posicionamiento del cuerpo. Conclusión: El uso de un simulador de baja fidelidad tuvo un impacto positivo en la promoción del conocimiento sobre la técnica de medición indirecta de la presión arterial en el hogar.

DESCRIPTORES: Presión Arterial, Determinación de la Presión Sanguínea, Educación en Enfermería, Materiales de Enseñanza, Simulación.

RECEBIDO EM: 03/15/2021 APROVADO EM: 06/10/2021

\section{Isabela Gomes Musa dos Santos}

Enfermeira Hospital Sírio-Libanês. Universidade de São Paulo, Escola de Enfermagem de Ribeirão Preto. Ribeirão Preto, SP, Brasil. ORCID: 0000-0002-6174-0155 


\section{Amanda dos Santos Oliveira}

Universidade de Araraquara, Araraquara, São Paulo, SP, Brasil.

ORCID: 0000-0002-2624-923X

\section{Ana Carolina Queiroz Godoy Daniel}

Hospital Israelita Albert Einstein, Unidade de Pronto Atendimento, São Paulo, SP, Brasil.

ORCID: 0000-0003-4877-9191

\section{Eugenia Veludo Veiga}

Universidade de São Paulo, Escola de Enfermagem de Ribeirão Preto. Ribeirão Preto, SP, Brasil.

ORCID: 0000-0003-3677-0210

\section{INTRODUÇÃO}

A hipertensão arterial (HA) é uma doença crônica não transmissível e o principal fator de risco para o desenvolvimento de doenças cardiovasculares. Estima-se que a doença atinja 36 milhões de pessoas no Brasil, com taxas de controle que não ultrapassam os $35 \%$. $^{(1)}$

A medida indireta da pressão arterial (PA) fora do ambiente do consultório é recomendada como estratégia para a elucidação diagnóstica da HA e para o acompanhamento e controle das cifras pressóricas; pode ser realizada pelo paciente ou pessoa capacitada para tal, com equipamento validado e calibrado, de maneira sistemática e normativa. ${ }^{(2)}$

As Diretrizes Brasileiras de Hipertensão Arterial - 2020 recomendam que o procedimento de medida indireta da PA deva ser realizado com técnica padronizada, em ambiente calmo e com temperatura agradável. ${ }^{(1)} \mathrm{O}$ paciente deve certificar-se que está com a bexiga vazia, que não praticou atividade física na última hora, não ingeriu bebida alcoólica ou cafeína e que não utilizou fumo ou tabaco nos últimos 30 minutos. Previamente à medida, deve posicionar-se de forma confortável e relaxada na cadeira, repousar o corpo por 5 minutos, manter as pernas descruzadas, os pés apoiados no chão, a palma da mão voltada para cima e o braço livre de roupas e na altura do coração. ${ }^{(1)}$

Embora a realização do procedimento no domicílio seja fortemente recomendada por diretrizes nacionais e internacionais, a escassez de estudos sobre o conhecimento do paciente quanto à técnica da medida indireta da PA evidencia importantes lacunas na educação em saúde e a necessidade de se desenvolver e imple- mentar intervenções eficazes, que possam garantir a execução adequada da técnica e a obtenção de valores fidedignos de PA. (3) Vale ressaltar que o conhecimento e a compreensão da população sobre a sua doença nem sempre é satisfatório, sendo este um ponto de dificuldade para adesão ao tratamento ou a inserção do usuário em seu plano de cuidados, a fim de evitar agravamentos e complicações. Cabe aos profissionais de saúde, por meio de estratégias de ensino propor atividades que ampliem o conhecimento dos usuários. ${ }^{(4)}$

$O$ uso da simulação, como parte integrante das metodologias ativas de ensino-aprendizagem, tem demonstrado resultados positivos na melhora do conhecimento do paciente sobre sua doença, aumento da adesão terapêutica, promoção da qualidade de vida e melhora de desfechos clínico ${ }^{(3,5-7)}$.

O simulador de baixa fidelidade é um protótipo estático, menos realístico, capaz de representar parcialmente a anatomia humana e permitir a réplica da técnica desejada. Quando utilizado como estratégia educativa pode colaborar com a apreensão do conhecimento, o desenvolvimento de habilidades e a melhora da autoconfiança na realização de procedimentos. Os simuladores vêm sendo amplamente utilizados na capacitação de profissionais e estudantes da área da saúde, bem como na educação de pacientes portadores de doenças crônicas que necessitam acompanhar sua condição de saúde e realizar procedimentos no domicílio. ${ }^{(8-10)}$

Acredita-se que a implementação de estratégias educativas com o uso de simulador de baixa fidelidade possa contribuir com a melhora do conhecimento de pa- cientes hipertensos sobre o procedimento de medida indireta da PA no domicílio. O presente estudo objetivou avaliar o conhecimento teórico-prático sobre a técnica da medida indireta da pressão arterial realizada por pacientes hipertensos no domicílio antes e após intervenção educativa com simulador de baixa fidelidade.

\section{MÉTODOS}

Trata-se de um estudo quase-experimental, do tipo antes e depois, que avaliou o conhecimento prático de pacientes portadores de HA sobre o procedimento de medida indireta da PA no domicílio antes e após a implementação de uma intervenção educativa com simulador de baixa fidelidade. A amostra de conveniência foi composta por pacientes hipertensos e maiores de 18 anos, abordados pelo pesquisador principal do estudo na sala de espera do posto de saúde, no horário que antecedia as consultas médicas. Foram excluídos pacientes que já haviam participado de qualquer outra estratégia de ensino sobre medida da PA, independente do hábito de medir a pressão no domicílio.

$\mathrm{O}$ estudo foi desenvolvido em uma Unidade de Estratégia de Saúde da Família, situada no município de Ribeirão Preto, no estado de São Paulo, durante os meses de janeiro a junho de 2016. Sendo aprovado pelo Comitê de Ética em Pesquisa com Seres Humanos da Escola de Enfermagem de Ribeirão Preto da Universidade de São Paulo, sob o parecer 0971/2008, conforme a Resolução 466/2012 do Conselho Nacional de Saúde.

No protocolo do estudo utilizou-se para a caracterização sócio demográfica e clínica 


\section{artigo}

Santos, I.G.N.; Oliveira, A.S.; Daniel, A.C.Q.G.; Veiga, E.V.

Ensino da medida da pressão arterial entre hipertensos: estratégia educativa com simulador de baixa fidelidade

um formulário de coleta de dados, desenvolvido por Santos, AS 2014(11), já utilizado em outro estudo voltado para a medida residencial da PA, composto pelos seguintes itens: gênero, idade, escolaridade, profissão, estado civil, hábito de aferir a PA, local em que realiza a aferição (no domicilio ou serviço de saúde) e diagnóstico médico referido de HA. A avaliação da medida indireta da PA se deu por meio do questionário intitulado "Conhecimento prático sobre a medida indireta da pressão arterial" desenvolvido e validado por Machado, JP 2014 (12). Tal questionário tem como finalidade

\section{Quadro1. Descrição dos itens 3,6 e 10-23 do questionário "Conhecimento prático sobre a medida indireta da pressão arterial"}

\section{PREPARO DO PACIENTE E AMBIENTE}

3. Prover ambiente calmo e silencioso

6. Orientar o paciente para não conversar durante a medida

10. Certificar-se de não haver bexiga cheia

11. Certificar-se de não haver prática de exercícios físicos 60 minutos antes

12. Certificar-se de não haver ingestão de bebidas alcoólicas 30 minutos antes

13. Certificar-se de não haver ingestão de café ou alimentos 30 minutos antes

14. Certificar-se de não haver fumado 30 minutos antes

\section{POSIÇÃO DO PACIENTE}

15. Manter pernas descruzadas

16. Manter os pés apoiados no chão quando sentado

17. Manter dorso recostado na cadeira

18. Manter corpo relaxado

19. Remover roupas do braço para colocar o manguito

20. Posicionar o braço na altura do coração

21. Manter o braço apoiado

22. Manter a palma da mão voltada para cima

23. Manter o cotovelo ligeiramente fletido

Figura 1- Simulador de baixa fidelidade, em formato de boneco articulado de madeira. Ribeirão Preto.2016

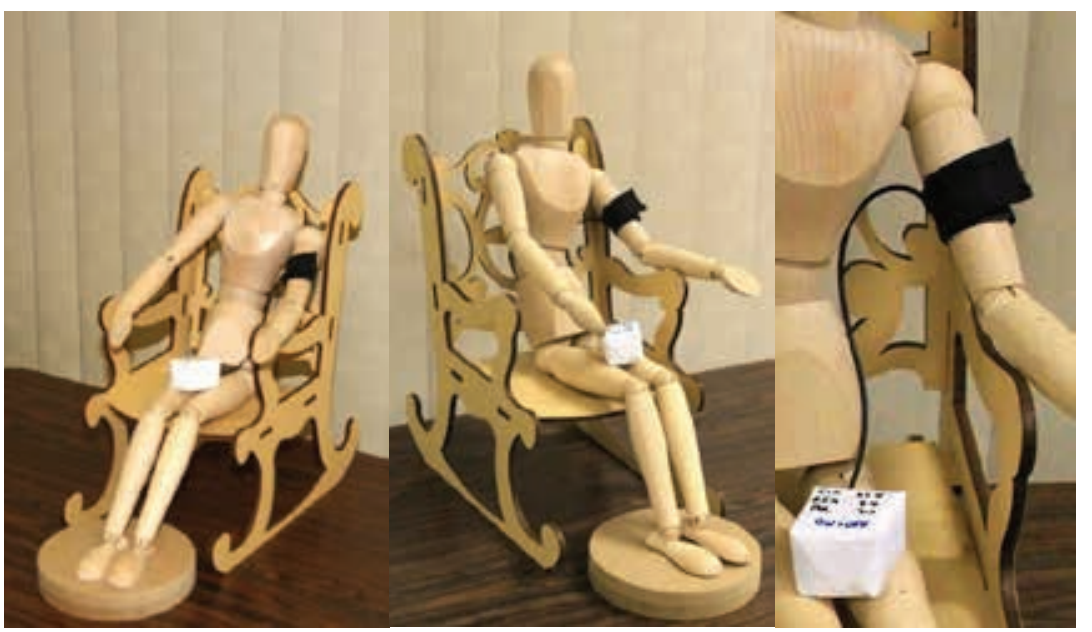

mensurar o conhecimento da medida indireta da PA entre profissionais de saúde; é composto por 49 itens, sendo 14 itens voltados ao preparo do paciente e ambiente, 8 itens sobre a posição do paciente, 16 itens sobre o procedimento de medida indireta da PA e 8 itens sobre o registro dos valores da PA. Trata-se de uma ferramenta já validada que inclui todas as etapas necessárias para a realização correta do procedimento e avalia o participante por meio de três opções de respostas: sim, não e não se aplica. Os itens três (3), seis (6) e 10 ao 23 foram selecionados para este estudo, pois estão relacionados às etapas "preparo do paciente e ambiente" e "posição do paciente", consideradas essenciais à realização da técnica no domicílio (Quadro 1).

O simulador de baixa fidelidade consistiu em um boneco de madeira, do tipo manequim, disponível para compra online, com tamanho de 51 centímetros e articulação nos ombros, cotovelos, mãos, dorso, quadril, joelhos e pés, composto por uma base circular do mesmo material, o qual o serve como sustentação, também incluída na simulação. (Figura 1) A escolha desse boneco se deu devido ao baixo custo e transporte fácil para a unidade de saúde onde foi aplicada a intervenção. As articulações do boneco de madeira permitiam seu posicionamento em uma cadeira de madeira com altura compatível ao apoio dos pés no chão, pernas descruzadas e palma da mão voltada para cima.

Para realizar a simulação, optou-se por desenvolver um aparelho oscilométrico e um manguito confeccionado de tecido e velcro que permitia envolver $40 \%$ do comprimento e $80 \%$ da largura do braço do boneco, para a simulação do aparelho foi utilizado papel cartão A4 e cola branca, material manufaturado de elaboração própria. (Figura 1). Também foram elaboradas placas ilustrativas contendo informações sobre o preparo do ambiente e do paciente previamente à realização da técnica de medida da PA - manter ambiente calmo, estar com bexiga vazia, manter repouso de pelo menos cinco minutos, aguardar 30 minutos quando tiver realizado atividade física ou ter consumido bebidas com cafeína ou álcool. (Figura 2) 
Figura 2 - Placas ilustrativas contendo informações sobre o preparo do ambiente e do paciente previamente à realização da técnica de medida da PA. Ribeirão Preto 2016.

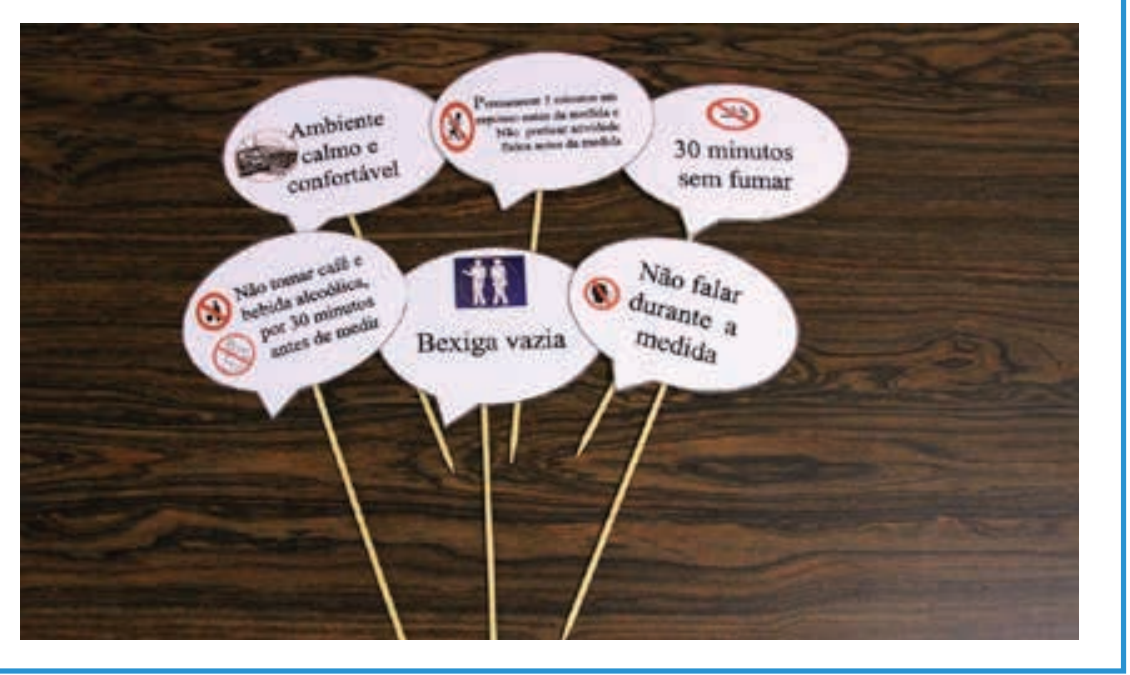

A intervenção educativa foi elaborada pelos pesquisadores do estudo e validada por cinco especialistas em HA e medida indireta da PA, que avaliaram a estratégia elaborada quanto a sua semântica, sintaxe, aparência e conteúdo. A validação da estratégia ocorreu durante uma reunião de pesquisa, momento em que os especialistas avaliaram os objetivos do estudo, o atendimento ao rigor do protocolo de medida da PA e o produto confeccionado (boneco de madeira, cadeira de madeira, aparelho de PA de tecido, manguito de tecido e placas ilustrativas) quanto à elegibilidade e a clareza.

O paciente foi convidado a participar do estudo e, em caso de aceite, foi submetido à intervenção educativa, com duração aproximada de 20 minutos; período em que foi orientado quanto aos objetivos, riscos e benefícios do estudo, e solicitado a assinar o Termo de Consentimento Livre e Esclarecido (TCLE). A abordagem individual ocorreu no momento da pré consulta no ambulatório de cardiologia, onde foi questionado sobre o diagnóstico autorreferido de HA, o hábito de medir a PA no domicílio e se já havia passado por alguma intervenção educativa na unidade. $\mathrm{O}$ participante que atendeu aos critérios de inclusão do estudo foi questionado quanto aos seus dados sociodemográficos e solicitado a demonstrar como realizava o posicionamento do seu corpo e braço durante o procedimento de medida da PA em sua residência utilizando o simulador de baixa fidelidade. Também foi solicitado ao participante que escolhesse as placas ilustrativas que julgasse necessárias para o preparo do ambiente antes da realização da medida da PA.

No período pré-intervenção, o instrumento de coleta de dados foi preenchido pelo pesquisador principal do estudo enquanto observava o posicionamento do boneco e a escolha das placas ilustrativas pelo participante. Após esta etapa, uma orientação verbal guiada pelo checklist de coleta dos dados e realizada por pesquisadores previamente treinados foi exposta aos participantes do estudo. A intervenção foi conduzida junto ao posicionamento do protótipo e explicada item a item para toda amostra. Tal intervenção seguiu com rigor as recomendações das Diretrizes Brasileiras de Monitorização Residencial da Pressão Arterial (MRPA) e das Diretrizes Brasileiras de Hipertensão Arterial.(1,2) No período pós-intervenção o cenário foi desmontado e o pesquisador avaliou o participante utilizando as mesmas etapas do procedimento pré-intervenção .

Os dados obtidos no instrumento de coleta de dados foram inseridos em banco de dados, imputados no programa Microsoft Office Excel com dupla digitação, e analisados no programa SPSS, versão 22. As variáveis descritivas foram analisadas por meio de frequências absolutas e relativas, e o teste de McNemar foi utilizado para comparar o conhecimento dos participantes antes e após a intervenção educativa. O nível de significância adotado foi de $5 \%(\alpha=0,05)$.

\section{elaborada quanto}

a sua semântica,

sintaxe, aparência e

conteúdo.

\section{RESULTADOS}

Participaram do estudo 30 pacientes com diagnóstico de HA, idade média de 58 anos, maioria do sexo feminino $(76,7 \%)$, com diferentes níveis de escolaridade e predominância do $1^{\circ}$ grau incompleto (50,0\%). A Tabela 1 apresenta as caracterís- 


\section{artigo}

Santos, I.G.N.; Oliveira, A.S.; Daniel, A.C.Q.G.; Veiga, E.V.

Ensino da medida da pressão arterial entre hipertensos: estratégia educativa com simulador de baixa fidelidade

Tabela 1- Caracterização dos participantes quanto a sexo, escolaridade e hábito de medir a pressão arterial ( $N=30)$, Ribeirão Preto, Brasil, 2016.

\begin{tabular}{|c|c|c|}
\hline CARACTERÍSTICAS DOS PARTICIPANTES & $\mathbf{N}$ & $\%$ \\
\hline \multicolumn{3}{|l|}{ Sexo } \\
\hline Feminino & 23 & 76,7 \\
\hline Masculino & 7 & 23,3 \\
\hline \multicolumn{3}{|l|}{ Escolaridade } \\
\hline $1^{\circ} \mathrm{Grau}$ Completo & 3 & 10,0 \\
\hline $1^{\circ}$ Grau Incompleto & 15 & 50,0 \\
\hline $2^{\circ}$ Grau Completo & 4 & 13,3 \\
\hline $2^{\circ} \mathrm{Grau}$ Incompleto & 8 & 26,7 \\
\hline \multicolumn{3}{|l|}{ Hábito de Medir a PA } \\
\hline $\operatorname{Sim}$ & 27 & 90,0 \\
\hline Não & 3 & 10,0 \\
\hline
\end{tabular}

Tabela 2- Frequência de acertos dos participantes nos itens 10-14 do questionário "Conhecimento prático sobre a medida indireta da pressão arterial", nos períodos pré e pós-intervenção(N=30), Ribeirão Preto, Brasil, 2016.

\begin{tabular}{|c|c|c|c|c|c|}
\hline \multirow[t]{2}{*}{ ITENS DO QUESTIONÁRIO } & \multicolumn{2}{|c|}{$\begin{array}{c}\text { PRÉ- } \\
\text { INTERVENÇÃO }\end{array}$} & \multicolumn{2}{|c|}{$\begin{array}{c}\text { PÓS- } \\
\text { INTERVENÇÃO }\end{array}$} & \multirow[t]{2}{*}{ P VALOR } \\
\hline & $\mathbf{N}$ & $\%$ & $\mathbf{N}$ & $\%$ & \\
\hline Prover ambiente calmo e silencioso & 18 & 60,00 & 26 & 86,67 & 0,008 \\
\hline $\begin{array}{l}\text { Orientar a não conversar durante a } \\
\text { medida }\end{array}$ & 22 & 73,33 & 28 & 93,33 & 0,016 \\
\hline $\begin{array}{l}\text { Certificar-se de não haver: bexiga } \\
\text { cheia }\end{array}$ & 18 & 60,00 & 26 & 86,67 & 0,021 \\
\hline $\begin{array}{l}\text { Certificar-se de não haver prática de } \\
\text { exercícios físicos } 60 \text { min antes }\end{array}$ & 21 & 70,00 & 27 & 90,00 & 0,070 \\
\hline $\begin{array}{l}\text { Certificar-se de não haver ingestão } \\
\text { bebidas alcoólicas } 30 \text { minutos antes }\end{array}$ & 13 & 56,67 & 27 & 90,00 & 0,000 \\
\hline $\begin{array}{l}\text { Certificar-se de não haver ingestão } \\
\text { de café ou alimentos } 30 \text { min antes }\end{array}$ & 12 & 40,00 & 26 & 86,67 & 0,000 \\
\hline $\begin{array}{l}\text { Certificar-se de não haver fumado } 30 \\
\text { minutos antes }\end{array}$ & 12 & 40,00 & 28 & 93,33 & 0,000 \\
\hline
\end{tabular}

Tabela 3- Frequência de acertos dos participantes nos itens 15-23 do questionário "Conhecimento prático sobre a medida indireta da pressão arterial", nos períodos pré e pós-intervenção (N=30), Ribeirão Preto, Brasil, 2016.

\begin{tabular}{lcccccc} 
& \multicolumn{3}{c}{ PRÉ- } & \multicolumn{2}{c}{ PÓS- } \\
ITENS DO QUESTIONÁRIO & \multicolumn{2}{c}{ INTERVENÇÃo } & \multicolumn{2}{c}{ INTERVENÇÃo } & P VALOR* \\
& N & $\%$ & N & $\%$ & \\
Manter pernas descruzadas & 27 & 90,00 & 30 & 100,00 & 0,250 \\
$\begin{array}{l}\text { Manter os pés apoiados no chão } \\
\text { quando sentado }\end{array}$ & 21 & 70,00 & 28 & 93,33 & 0,016 \\
\end{tabular}

ticas dos participantes quanto a sexo, escolaridade e o hábito de medir a PA.

A Tabela 2 mostra a frequência de acertos dos participantes nos períodos pré e pós-intervenção, quando avaliados sobre a etapa "preparo do paciente e ambiente".

A Tabela 3 mostra a frequência de acertos dos participantes, nos períodos pré e pós-intervenção, quando avaliados sobre a etapa "posição do paciente".

Os resultados evidenciaram que nenhum dos participantes do estudo realizou as etapas do procedimento com $100 \%$ de acertos antes da intervenção educativa. Dos 27 participantes que referiram o hábito de realizar a medida da PA fora do ambiente de consultório seis realizaram o procedimento da maneira adequada, com $100 \%$ de acertos, no período pós-intervenção.

\section{DISCUSSÃO}

Este estudo buscou avaliar o conhecimento prático de pacientes hipertensos sobre o procedimento de medida indireta da PA no domicílio, antes e após a implementação de uma intervenção educativa com simulador de baixa fidelidade.

A obtenção de valores fidedignos de PA por meio da medida indireta perpassa por etapas essenciais que norteiam o procedimento a ser realizado de forma padronizada e segura, conforme a recomendação de diretrizes nacionais e internacionais. ${ }^{(1,2,13)}$ Evidências da literatura têm demonstrado que tanto os estudantes quanto os profissionais de saúde, com longo tempo de formação e experiência prática, enfrentam dificuldades na execução do procedimento devido à mecanização do cuidado e à falta de conhecimento e de habilidades sobre a técnica. ${ }^{(8,14-16)}$ Intervenções educativas que utilizam estratégias de ensino aprendizagem tem sido desenvolvidas, nos ambientes assistenciais e acadêmicos, na tentativa de suprir as fragilidades encontradas na execução da medida. Os resultados dessas intervenções mostraram que o uso de jogos educativos e de ambiente de simulação realística contribuiu para o desenvolvimento de habilidades técnicas, para o estabelecimento de práticas segu- 


\begin{tabular}{|c|c|c|c|c|c|}
\hline $\begin{array}{l}\text { Manter o dorso recostado na } \\
\text { cadeira }\end{array}$ & 11 & 63,33 & 27 & 90,00 & 0,000 \\
\hline Manter o corpo relaxado & 8 & 26,67 & 28 & 93,33 & 0,000 \\
\hline $\begin{array}{l}\text { Posicionar o braço na altura do } \\
\text { coração }\end{array}$ & 6 & 20,00 & 27 & 90,00 & 0,000 \\
\hline Manter o braço apoiado & 8 & 26,67 & 27 & 90,00 & 0,000 \\
\hline $\begin{array}{l}\text { Manter a palma da mão voltada } \\
\text { para cima }\end{array}$ & 15 & 50,00 & 27 & 90,00 & 0,000 \\
\hline Cotovelo fletido & 12 & 40,00 & 28 & 93,33 & 0,000 \\
\hline * Nota: P valor teste de Mc Nemar & & & & & \\
\hline
\end{tabular}

ras e para a promoção do conhecimento profissional. ${ }^{(11,12)}$ Quando analisado o conhecimento do paciente sobre HA, independentemente do nível de escolaridade, estudos revelaram que os hipertensos conhecem as informações essenciais sobre a doença e os valores de referência para o controle a das cifras pressóricas, entretanto desconhecem as complicações e agravos da HA, bem como suas implicações para a saúde cardiovascular. ${ }^{(17,18)}$

A medida da PA no domicílio tem a finalidade de contribuir com o monitoramento e com controle da HA. A aquisição de aparelhos automáticos oscilométricos tem sido cada vez mais recomendada para uso residencial, uma vez que esses equipamentos são portáteis, de fácil utilização, baixo custo e podem ser facilmente encontrados em farmácias, casas de materiais médico-cirúrgicos e lojas virtuais. Os aparelhos oscilométricos possuem algoritmos pré-programados que calculam automaticamente os valores de PA com precisão, além de permitir o armazenamento de valores de PA e de frequência cardíaca em medidas consecutivas. Sendo que o uso de tais aparelhos para monitoramento de saúde entre idosos vêm crescendo ultimamente, ainda que sem treinamento para a sua melhor utilização e obtenção de valores fidedignos. ${ }^{(11-19)}$

Os resultados do presente estudo mostraram que o conhecimento do hipertenso sobre o procedimento de medida indireta da PA melhorou, significativamente, após a aplicação da intervenção educativa, em todos as variáveis analisadas, referentes ao "preparo do paciente e ambiente" e "posição do paciente". Acredita-se que o uso de um simulador de baixa fidelidade tenha permitido

\section{A aquisição \\ de aparelhos \\ automáticos \\ oscilométricos tem}

sido cada vez mais

recomendada para

uso residencial,

uma vez que esses

equipamentos são

portáteis, de fácil

utilização, baixo

custo e podem

ser facilmente

encontrados em

farmácias, casas de

materiais médico-

cirúrgicos e lojas

virtuais. maior interesse dos sujeitos no processo de ensino-aprendizagem, visto o fácil manuseio do boneco de madeira, a possibilidade de repetição da técnica e a aproximação da técnica com aspectos da realidade.

A educação em saúde é campo de diversos estudos experimentais e quase-experimentais, que se propóem a comparar o efeito de intervenções com diferentes estratégias e métodos de ensino-aprendizagem, tais como: vídeos educativos, acompanhamento telefônico, cartilhas educativas, jogos educacionais, softwares e aplicativos em saúde. O ensino por simulação é uma estratégia de ensino-aprendizagem que permite estimular a tomada de decisão frente as situações vivenciadas pelo usuário, ampliando o contato e o conhecimento com o problema em questão. A transmissão de conhecimento aos pacientes tem alto impacto quando associados a estratégias educativas que englobem a metodologia ativa, pois mostram efeitos positivos para ampliar a confiança dos usuários na resolução de conflitos e obtenção do conhecimento. $\quad(11,20,21)$

Os resultados apresentados neste estudo comprovaram que a implementação de uma estratégia educativa utilizando simulador de baixa fidelidade pode melhorar o conhecimento do paciente hipertenso quanto à realização correta da medida residencial da PA. Além do mais, podem contribuir no controle da HAS, assim auxiliando na prevenção em saúde de seus usuários. ${ }^{(17,22)}$

\section{CONCLUSÕES}

A implementação de uma estratégia educativa utilizando simulador de baixa fidelidade mostrou-se efetiva na promoção do conhecimento prático sobre a técnica da medida indireta da PA na residência entre pacientes hipertensos. As limitações desta pesquisa relacionam-se ao pequeno número de participantes e à utilização de uma amostra de conveniência proveniente de um único centro de saúde, o que impossibilita a generalização dos resultados obtidos. Ademais, a pesquisa é de caráter transversal e não permite a avaliação posterior do conhecimento prático adquirido 


\section{artigo}

pelos pacientes. Diante deste contexto, sugere-se o desenvolvimento de estudos experimentais, prospectivos, com amostras representativas, com emprego de simula- dores que possam ser utilizados em diferentes ambientes de assistência à saúde.

Acredita-se que a aplicação de estratégias inovadoras de ensino-aprendizagem, de bai- xo custo e fácil transporte, possam contribuir com a educação em saúde, com a gestão do autocuidado e com o controle dos valores de pressão arterial entre hipertensos. I.

\section{REFERÊNCIAS}

1. Brasil. Lei n. 8.080 de 19 de setembro de 1990. Lei Orgânica da Saúde. Diário Oficial da União, Brasília, DF, p. 18.055, 20 set. 1990.

2. Brasil. Ministério da Saúde. Decreto 7.508 de 28 de junho de 2011. Regulamenta a Lei $n^{\circ} 8.080$ de 19 de setembro de 1990. Diário Oficial da União, Seção 1, 29 jun. 2011.

3. Brasil. Portaria GM/MS no 4.279, de 30 de dezembro de 2010. Estabelece diretrizes para a organização da Rede de Atenção à Saúde no âmbito do SUS. Brasília: Ministério da Saúde; 2010.

4. Zilli MG, Santos APS, Yamaguchi CK, Borges LA. Contribuições de uma clínica escola no atendimento em saúde no Sul de Santa Catarina. RIES. 2017; 6(1):105-116.

5. Fernandes LFB, Silvares EFM, Miyazaki MCOS. Caracterização da população atendida em ambulatório de Psicologia da Saúde de um hospital-escola. Contextos Clínic. 2017;10(2):145-156.

6. Ribeiro MMF, Amaral CFS. Medicina centrada no paciente e ensino médico: a importância do cuidado com a pessoa e o poder médico. Rev. bras. educ. med. 2008; 32(1):90-97.

7. Chazan ACS, Silveira LMC, Favoreto CAO. Revisão de prontuário como estratégia de ensino-aprendizagem da medicina centrada na pessoa em um ambulatório universitário no município do Rio de Janeiro. Rev Bras Med Fam Comunidade. 2014; 9(30):96-103.

8. Brasil. Ministério da Educação. Conselho Nacional de Educação, Câmara de Educação Superior. Resolução CNE/CES n% de 7 de novembro de 2001. Institui diretrizes curriculares nacionais do curso de graduação em Medicina. Diário Oficial da União. Brasília, 9 nov. 2001; Seção 1, p.38.

9. Santos ML, Novaes CO, Iglesias AC. Epidemiological profile of patients seen in the pre-anesthetic assessment clinic of a university hospital. Rev Bras Anestesiol. 2017; 67(5): 457-467.

10. Guedes BAP, Vale FLB, Souza RW, Costa MKA, Batista SR. A Organização Da Atenção Ambulatorial Secundária na SESDF. Ciênc. saúde colet. 2019; 24(6):2125-2134.

11. Vasconcelos GMT, Santos GKBB, Santos ACFS, Silva JOM, Santos ES. Perfil sociodemográfico dos pacientes atendidos em um ambulatório público de cuidados com feridas complexas. Anais Congresso Internacional de Enfermagem; 2019; Sergipe, Unit, 2019.

12. Pexe M, Carvalho DLBC, Menotti AFS, Soares JGS, Jardim NA, Casalenuovo PRM. Perfil epidemiológico do Ambulatório de Saúde Mental Infantojuvenil da Clínica Integrada do Centro Universitário de Várzea Grande (UNIVAG). Caderno de Publicações Univag. 2019; 10:4-14.

13. Adamczyk SP, Souza SJP. Perfil epidemiológico dos pacientes atendidos no ambulatório de enfermagem. Rev Gestão e Saúde. 2018; 19(1): 118-28.

14. Dantas MM, Solá ALN, Pires DAL, Silva JBP, Reis MAC, Herrera MR, Herrera SDSC. Profile of Patients Attended in the Cardiology
Ambulatory of the South Region of Tocantins in the Year 2015. Revista Cereus. 2019; 11(2):1-11.

15. Hodniki PP, Teixeira CRS, Zanetti ML, Moraes C, Fermino RC, Berra S. Prática de atividade física e ambiente percebido de usuários do Sistema Único de Saúde. Saúde coletiva. 2021;11(65): 6280-87.

16. Brasil. Pesquisa Nacional de Saúde: 2013: acesso e utilização dos serviços de saúde, acidentes e violências: Brasil, grandes regiões e unidades da federação / IBGE, Coordenação de Trabalho e Rendimento - Rio de Janeiro: IBGE, 2015.

17. Sena NV, Alves AFV, Pontes LM, Silva PRS, Duffles MSL, Souza MLP, Brandão BJF. Perfil epidemiológico dos pacientes atendidos no serviço de dermatologia da BWS. BWS Journal. 2020; 3:1-9.

18. Saraiva LGF, Dornelas PG, Cau SBA, Calábria LK. Perfil epidemiológico de pacientes atendidos em uma rede ambulatorial do Hiperdia Minas em Governador Valadares-MG. Revista de Atenção à Saúde. 2016; 14(48): 40-47.

19. Viacava F, Porto SM, Carvalho CC, Bellido JG. Desigualdades regionais e sociais em saúde segundo inquéritos domiciliares (Brasil, 1998-2013). Ciênc. saúde colet. 2019; 24(7): 2745-60.

20. Franco RJS. A atividade física no presente pode ser a receita para evitar os males da obesidade e hipertensão no futuro. Arq. Bras. Cardiol. 2020; 15(1): 50-51.

21. Lemos LN, Tavares RMF, Donadelli CAM. Perfil epidemiológico de pacientes com colelitíase atendidos em um ambulatório de cirurgia. Revista Eletrônica Acervo Saúde/ Electronic Journal Collection Health. 2019;28(28):1-9.

22. Silva LCC, Araújo AJ, Queiroz AMD, Sales MPU, Castellano MVCO. Controle do tabagismo: desafios e conquistas. J. bras. pneumol. 2016; 42(2): 290-298.

23. Brasil. Ministério do Trabalho e Emprego - MTE. Relação Anual de Informações Sociais - RAIS, 2013. Disponivel em <http://www. mte.gov.br/rais/default.asp>. Acesso em: 28 jul. 2020.

24. Gouveia N, Leon AP, Junger W, Lins JF, Freitas CU de. Poluição do ar e impactos na saúde na Região Metropolitana de Belo Horizonte - Minas Gerais, Brasil. Ciênc. saúde colet. 2019; 24(10): 3773-81.

25. Lemos AP, Ribeiro C, Fernandes J, Bernardes K, Fernandes R. Saúde do homem: Os motivos da procura dos homens pelos serviços de saúde. Rev. enferm. UFPE online. 2017; 11(11): 4546-53.

26. Sousa RJ, Soares TM, Rosario CR, Rosa DPS, David RAR, Brito HES. Aspectos da masculinidade como impeditivo do autocuidado na saúde do homem. Saúde coletiva. 2021; 11(65): 6306-6314.

27. Soares MOM, Higa EFR, Passos AHR, Ikuno MRM, Bonifácio LA, Mestieri CP, Ismael RK. Reflexões contemporâneas sobre anamnese na visão do estudante de Medicina. Rev. bras. educ. med. 2014; 38(3): 314-22. 